\title{
Silencing of perilipin by short hairpin RNA inhibits proliferation and induces apoptosis in liposarcoma cells
}

\author{
LING-XIN MENG ${ }^{1}$, YU-XIU ZHENG ${ }^{1}$, MAO-LEI HE ${ }^{1}$, XIAO-MING ZHOU ${ }^{1}$, SHU-YAN SUN ${ }^{2}$, \\ ZHAO-JUN DING ${ }^{1}$, QIN MENG ${ }^{1}$, BING-CHENG LI ${ }^{1}$ and YAN-WEI SUN ${ }^{1}$ \\ Departments of ${ }^{1}$ Oncology and ${ }^{2}$ Pathology, People's Hospital of Rizhao, Rizhao, Shandong 276826, P.R. China
}

Received September 27, 2017; Accepted May 18, 2018

DOI: $10.3892 / \mathrm{mmr} .2018 .9461$

\begin{abstract}
Previous studies have identified that perilipin-1 (PLIN1) is a highly specific marker for liposarcoma. However, its functions have yet to be fully elucidated. The aim of the present study was to investigate the potential role of PLIN1 in the proliferation, migration and apoptosis of liposarcoma cells. Short hairpin RNA was designed to inhibit PLIN1 levels. Cell proliferation was monitored by Cell Counting Kit- 8 assay and cell migration determined by wound healing assay. Flow cytometry was performed to assess the cell cycle distributions and apoptosis in liposarcoma cells. The results demonstrated that the expression of PLIN1 was significantly upregulated in liposarcoma tumor tissues compared with normal adipose tissues. Silencing of PLIN1 by short hairpin RNA significantly inhibited proliferation and migration and induced $G_{1}$ phase cell cycle arrest and apoptosis in liposarcoma cell lines. It was identified that PLIN1 serves a crucial role in the pathogenesis and progression of liposarcoma and may be a potential therapeutic target for its clinical management.
\end{abstract}

\section{Introduction}

Liposarcoma is the most frequently occurring soft-tissue tumor and accounts for $>20 \%$ of all soft tissue sarcomas $(1,2)$. Liposarcoma has been classified into four major histological sub-types: Well-differentiated liposarcoma, dedifferentiated liposarcoma, myxoid liposarcoma and pleomorphic liposarcoma (3). The 5-year survival rate is affected by different sub-types, with $75-100 \%$ in differentiated tumors. However, pleomorphic liposarcoma has the worst 5-year survival rate of $0-20 \%$ (4). In clinical practice, surgical approaches are first-line therapy in the management of liposarcoma, accompanied with radiotherapy and chemotherapy (5). Currently, there is no therapeutic option for aggressive and metastatic

Correspondence to: Dr Ling-Xin Meng, Department of Oncology, People's Hospital of Rizhao, 126 Taian Road, Rizhao, Shandong 276826, P.R. China

E-mail: wanglingx11@aliyun.com

Key words: perilipin-1, liposarcoma, apoptosis, cell cycle arrest liposarcoma (5). Therefore, exploring novel and specific therapeutic targets for liposarcoma is important.

Perilipin-1 (PLIN1) is a member of the perilipin/APRP/ TIP47 (PAT) protein family and exists in at least 3 protein isoforms (molecular weight $\sim 56 \mathrm{kDa}$ ) (6). PLIN1 is a biomarker of adipocyte differentiation and involved in regulating lipid droplet biogenesis and hydrolysis (7). In $\mathrm{db} / \mathrm{db}$ mice, PLIN1 loss-of-function accelerates lipolysis and decreases the size of lipid droplets in adipocytes, causing resistance to diet-induced obesity (8). In addition, downregulation of PLIN1 expression is also associated with the acceleration of lipolysis in 3T3-L1 adipocytes (9). In humans, the expression of PLIN1 in adipose tissues is positively correlated with insulin sensitivity (10). Previous studies have revealed that PLIN1 can regulate spermatogenesis (11), atherosclerotic lesions (12) and osteogenic differentiation (13). Notably, PLIN1 is known to be an independent poor prognostic factor in metastatic breast cancer and can inhibit cell proliferation, migration, invasion and tumorigenesis in mice $(7,14)$. Only one study, to the best of the authors' knowledge, has examined the expression of PLIN1 in liposarcoma (6) but the association between PLIN1 and cell proliferation, migration and apoptosis has not been investigated in vitro.

The present study examined the expression of PLIN1 in liposarcoma tissues and identified that PLIN1 was significantly overexpressed. In addition, PLIN1 levels in liposarcoma cell lines were downregulated by short hairpin RNA (shRNA) and cell proliferation, migration and apoptosis experiments were performed to elucidate its function in vitro.

\section{Materials and methods}

Patients and specimens. A total of 12 pairs of liposarcoma tumor tissues and matched adjacent non-tumorous adipose tissues were collected from patients ( 8 males and 4 females; age range: $15-67$ years; mean age: $48.8 \pm 14.0$ years) who had undergone surgery at the Department of Oncology, People's Hospital of Rizhao (Rizhao, China) between January 2014 and June 2016. None of the patients had been subjected to preoperative radiotherapy or chemotherapy and all had been diagnosed with liposarcoma based on histopathological evaluation. All collected tissue samples were immediately stored in liquid nitrogen. Human samples were obtained with written informed consent from all patients. The study was approved 
by the Ethics Committee of the People's Hospital of Rizhao (Approval Number: KYLL-20140903).

Cell culture. T778 (well differentiated) and LPS141 (dedifferentiated) liposarcoma cell lines were purchased from the Cell Bank of China Academy of Sciences (Shanghai, China). The cell lines were cultured in RPMI medium 1640 (Invitrogen; Thermo Fisher Scientific, Inc., Waltham, MA, USA) supplemented with $10 \%$ fetal bovine serum (Gibco; Thermo Fisher Scientific, Inc.) and $1 \%$ penicillin-streptomycin at $37^{\circ} \mathrm{C}$ in $5 \%$ $\mathrm{CO}_{2}$ and were plated in a 6-well plate at a density of $2 \times 10^{5}$ cells per well. Following incubation for 2 days, cells were collected for proliferation, migration and apoptosis assays.

Cell counting kit (CCK)- 8 proliferation assay. The CCK- 8 proliferation assay was conducted as previously described (15). The proliferation of cells $\left(1 \times 10^{4}\right)$ was measured using a CCK-8 Cell Proliferation/Viability Assay kit (Dojindo Molecular Technologies, Inc., Kumamoto, Japan).

Construction of ShRNA-PLIN1 eukaryotic expression plasmid. shRNA was constructed to specifically target PLIN1 using shRNA design tools (http://rnaidesigner.thermofisher. com/rnaiexpress/). Using BLAST (http://blast.ncbi.nlm .nih.gov/Blast.cgi), it was verified that the designed shRNA targeted only the PLIN1. The shRNA was synthesized by Shanghai GenePharma Co., Ltd. (Shanghai, China). The target site in human gene encoding PLIN1 was: shRNA-PLIN1 sense strand, 5'-GGGGACACAGTGTGCATTA-3', shRNA-PLIN1 antisense strand, 5'-TGGCACATACCCTGCAGAAGA-3'; shRNA-Con sense strand, 5'-GCCCTCGGTGTCCTACTT CA-3', shRNA-Con antisense strand, 5'-ATTTGAAGTAGG ACACCGAGG-3'. Each DNA was used to transform the $E$. coli strain DH5 $\alpha$ and purified with a plasmid purification kit (Qiagen, Inc., Valencia, CA, USA). A total of $1 \mu \mathrm{g}$ plasmid was transfected into T778 and LPS141 cells using Lipofectamine ${ }^{\circledR}$ 2000 (Invitrogen; Thermo Fisher Scientifc, Inc.) for $48 \mathrm{~h}$ at $37^{\circ} \mathrm{C}$, as previously described (16). The infected cells were selected with puromycin $(2 \mathrm{mg} / \mathrm{ml}) 48 \mathrm{~h}$ following transfection. Then the clones were selected and cultured for further experimentation.

Immunohistochemical staining and western blotting. The tissues were fixed in $4 \%$ formaldehyde at room temperature for $24 \mathrm{~h}$ and embedded in paraffin. The paraffin-embedded tumor tissues and adipose tissues were cut into $\sim 3 \mu \mathrm{m}$ sections, which were dehydrated at $65^{\circ} \mathrm{C}$ for $24 \mathrm{~h}$, deparaffinized in xylene and rehydrated in a descending ethanol series. Subsequently, the sections were evaluated immunohistochemically using anti-human PLIN1 (cat. no. ab61682, dilution, 1:100; Abcam, Cambridge, UK), as previously described (17). Image visualization was performed under a microscope (Leica DM 2500; Leica Microsystems GmbH, Wetzlar, Germany) and the integrated optical density (IOD) was measured by Image Pro-Plus 6 software (Media Cybernetics, Inc., Rockville, MD, USA).

Protein was extracted using radioimmunoprecipitation assay lysis buffer (Beyotime Institute of Biotechnology, Haimen, China). The concentration was determined using the bicinchoninic acid kit for protein determination (Sigma-Aldrich; Merck KGaA, Darmstadt, Germany). Samples containing $50 \mu \mathrm{g}$ of protein were separated by $10 \%$ SDS-PAGE gel and transferred onto nitrocellulose membranes (Bio-Rad Laboratories, Inc., Hercules, CA, USA). The membranes were blocked with $10 \%(\mathrm{w} / \mathrm{v})$ non-fat dry milk in Tris-buffered saline containing $0.1 \%(\mathrm{w} / \mathrm{v})$ Tween 20 at room temperature for $2 \mathrm{~h}$. Primary antibody PLIN1 (cat. no. ab61682, dilution, 1:1,000; Abcam, Cambridge, UK) was incubated for $2 \mathrm{~h}$ at room temperature and membranes were washed three times (10 min/wash) in PBS. Next, the membranes were incubated with the appropriate horseradish peroxidase-conjugated secondary antibody (cat. no. sc-516102; dilution: 1:10,000; Santa Cruz Biotechnology, Inc., Dallas, TX, USA) at room temperature for $2 \mathrm{~h}$, and then visualized using SuperSignal ${ }^{\mathrm{TM}}$ West Pico PLUS Chemiluminescent Substrate (cat. no. 34580; Thermo Fisher Scientific, Inc.). Glyceraldehyde-3-phosphate dehydrogenase (GAPDH; cat. no. 2118; dilution: 1:2,000; Cell Signaling Technology, Inc., Danvers, MA, USA) was used as the control antibody. Signals were densitometrically assessed using Quantity One software version 4.5 (Bio Rad Laboratories, Inc.).

Wound healing assay. T778 and LPS141 cells $\left(2 \times 10^{5}\right)$ were trypsinized and reseeded in each well of a new 6-well plate. Following $24 \mathrm{~h}$ incubation at $37^{\circ} \mathrm{C}$ in $5 \% \mathrm{CO}_{2}$, the confluent cell monolayers were scratched with a $10 \mu \mathrm{l}$ sterile pipette tip. Then the non-adherent cells were washed off with sterilized PBS and serum-free medium was added to the wells. The gap area caused by the scratch was observed using an inverted microscope (Olympus Corporation, Tokyo, Japan). Images were captured from 3 random non-overlapping areas in each well at $12 \mathrm{~h}$ post-scratch. The scratch width between the two linear regions was measured to assess the capacity of cells to migrate.

Flow cytometry for the detection of cell cycle progression and apoptosis. T778 and LPS141 cells were collected following digestion and were washed twice with PBS and centrifuged at $1,200 \mathrm{x} \mathrm{g}$ for $5 \mathrm{~min}$ at $4^{\circ} \mathrm{C}$. The supernatant was discarded and the cells resuspended and fixed in ice-cold $75 \%$ ethanol then stored at $4^{\circ} \mathrm{C}$. Following two washes in PBS, the cells were stained with propidium iodide (PI; cat. no. F10797; Invitrogen; Thermo Fisher Scientific, Inc.) at $4^{\circ} \mathrm{C}$ for $30 \mathrm{~min}$ in the dark and acquired on a flow cytometer. Flow cytometric analysis of the percentage of cells in $\mathrm{G}_{1}, \mathrm{~S}$ and $\mathrm{G}_{2}$ phases (BD Biosciences, Franklin Lakes, NJ, USA) was performed. The data were processed by Cell Quest Software (version 5.1, BD Biosciences). The cell apoptosis assay was determined as previously described (15). The Annexin V-FITC apoptosis detection kit was purchased from Invitrogen (Thermo Fisher Scientific, Inc.).

Statistical analysis. The experiments were repeated three times and the data are presented as the means \pm standard deviation for each group. All statistical analyses were performed using PRISM version 7.0 (GraphPad Software, Inc., La Jolla, CA, USA). Student's t-test was used to analyze two-group differences. Inter-group differences were analyzed by one-way analysis of variance, followed by a post hoc Tukey's test for multiple comparisons. $\mathrm{P}<0.05$ was considered to indicate a statistically significant difference. 


\section{Results}

PLIN1 is overexpressed in liposarcoma tissues compared with normal adipose tissue. Immunohistochemical staining was performed in normal control and liposarcoma tumor tissues, and the results demonstrated that PLIN1 expression increased in liposarcoma tumor tissues, revealing a statistically significant difference compared with normal control (Fig. 1A and B). To further confirm PLIN1 expression, western blotting analysis was performed and identified that PLIN1 protein expression was significantly upregulated in liposarcoma tumor tissues compared with the control group (Fig. 1C and D). These findings validated the hypothesis that PLIN1 is closely associated with liposarcoma.

Knockdown of PLIN1 inhibits proliferation of liposarcoma cells. To investigate its function in vitro, shRNA to inhibit the levels of PLIN1 was designed. As illustrated in Fig. 2A, the levels of PLIN1 were significantly inhibited by shRNA-PLIN1 in T778 and LPS141 cells compared with the negative control (Negative-Con) or shRNA-Con group. No significant difference was observed in the levels of PLIN1 between the Negative-Con and shRNA-Con group. Next, the proliferation of T778 and LPS141 cells following transfection with shRNA-PLIN1 was analyzed using the CCK-8 assay. The results revealed that the proliferation of T778 and LPS141 cells was markedly suppressed by PLIN1 depletion compared with the Negative-Con or shRNA-Con group at days 3 and 5 (Fig. 2B). No significant difference was observed in the proliferation curves of Negative-Con and shRNA-Con transfected into T778 or LPS141 cells. These data indicated that PLIN1 loss-of-function inhibited the proliferation of liposarcoma cells.

Knockdown of PLIN1 inhibits migration of liposarcoma cells. The effect of PLIN1 knockdown on cell migration was determined by a wound healing assay. T778 and LPS141 cells were seeded, and then scratches made $24 \mathrm{~h}$ later. Transfection of shRNA-PLIN1 led to retarded wound closing compared with the Negative-Con or shRNA-Con groups in T778 and LPS141 cells for $12 \mathrm{~h}$ (Fig. 3A and B). However, no significant difference was observed in the wound closing results between the Negative-Con and shRNA-Con groups in T778 and LPS141 cells (Fig. 3A and B). These findings indicated that PLIN1 loss-of-function inhibited the migration of liposarcoma cells.

Knockdown of PLIN1 induces cell cycle arrest and apoptosis of liposarcoma cells. Flow cytometry analysis was performed to further evaluate whether silencing of PLIN1 regulates liposarcoma cell proliferation by altering the progression of cell cycle and apoptosis. T778 and LPS141 cells were transfected with Negative-Con, shRNA-Con or shRNA-PLIN1 for $24 \mathrm{~h}$, and cell cycle distributions were determined by PI staining. As presented in Fig. 4A and B, transfection with shRNA-PLIN1 led to increase of cells in the $G_{1}$ phase and decrease of cells in the $\mathrm{S}$ phase, while transfection with shRNA-PLIN1 had no evident effect on the $G_{2}$ phase of the cell cycle in T778 and LPS141 cells. In addition, no significant difference was observed in cell cycle distribution between Negative-Con and shRNA-Con transfected into T778 or LPS141 cells. The rate of apoptosis was significantly increased when T778 and LPS141 cells were transfected with shRNA-PLIN1 compared with the Negative-Con or shRNA-Con group (Fig. 5A and B). These findings indicated that PLIN1 loss-of-function could induce $\mathrm{G}_{1}$ phase cell cycle arrest and apoptosis in T778 and LPS141 cells. Based on aforementioned findings, it was demonstrated that knockdown of PLIN1 inhibited the proliferation of liposarcoma cells, at least partly, by inducing $\mathrm{G}_{1}$ phase cell cycle arrest and apoptosis.

\section{Discussion}

The present study demonstrated robust protein expression of PLIN1 in liposarcoma tumor tissues. Silencing of PLIN1 inhibited the proliferation and migration of liposarcoma cells. In addition, $\mathrm{G}_{1}$ phase cell cycle arrest and apoptosis were induced by the knockdown of PLIN1 in liposarcoma cells. These data suggested that upregulation of PLIN1 may serve an oncogenic role in the pathogenesis and progression of liposarcoma. Therefore, it is hypothesized that PLIN1 may be a potential therapeutic target for the clinical management of liposarcoma.

PLIN1 is the major lipid droplet-coating protein of mature adipocytes and has a high specificity for adipocytes of white and brown fatty tissue $(18,19)$. PLIN1 is known to be highly expressed in Leydig cells and is closely associated with steroidogenesis and spermatogenesis (11). Straub et al (20) demonstrated that PLIN1 is present in hepatocellular carcinoma, sebaceous tumors and mesenchymal tumors with adipogenic differentiation, but PLIN1 is restricted to lipid droplets in tumors. The other members of the PAT protein family, adipocyte-differentiation-related protein (adipophilin, PLIN2) and tail-interacting protein of $47 \mathrm{kDa}$ (TIP47, PLIN3), are almost ubiquitously expressed in lipid droplets in various tumor types and normal tissues $(20,21)$. Westhoff et al (6) demonstrated that more than two-thirds of liposarcomas present PLIN1 positivity and that all non-lipomatous sarcomas studied are negative for this marker, suggesting that PLIN1 is a highly specific marker for liposarcoma. The present study demonstrated that PLIN1 protein expression was significantly upregulated in liposarcoma tumor tissues, and this was verified by immunohistochemical staining and western blotting. These findings suggested that robust expression of PLIN1 may possess a potential role in the pathogenesis and development of liposarcoma.

Increasing evidence suggests that PLIN1 is positively associated with poor overall survival rates in metastatic breast cancer and lung cancer $(7,14)$. Zhou et al (7) demonstrated that PLIN1 mRNA expression is significantly downregulated in human breast cancer, and that the overexpression of PLIN1 in MCF-7 and MDA-MB-231 cells markedly suppresses cell proliferation, migration, invasion and in vivo tumorigenesis in mice. Conversely, the present study identified that PLIN1 was significantly increased in liposarcoma tumor tissues and that downregulation of PLIN1 by shRNA in T778 (well differentiated liposarcoma cell line) and LPS141 (dedifferentiated liposarcoma cell line) suppressed cell proliferation and migration. These findings suggested that PLIN1 expression may be tissue-specific. A previous study indicated that proliferation inhibition in liposarcoma cells may be a result of cell cycle 
A

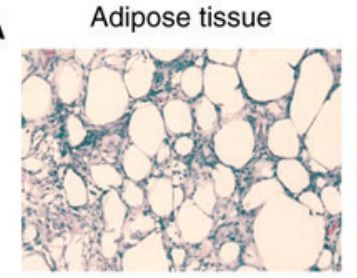

Liposarcoma

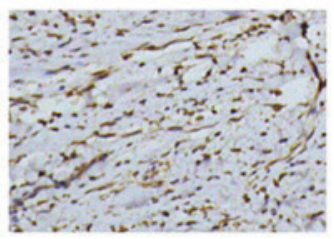

C

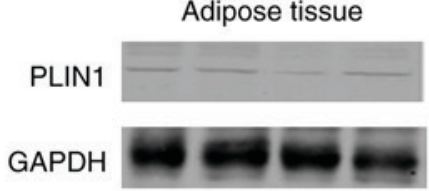

D

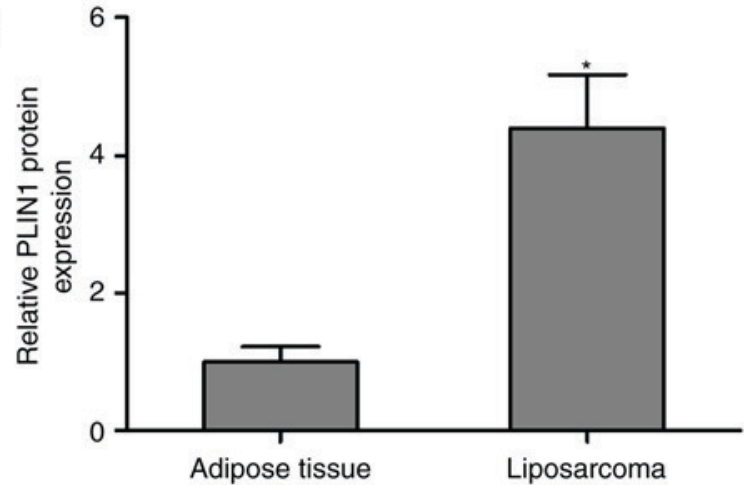

Figure 1. Protein expression of PLIN1 in liposarcoma tumor tissues and normal adipose tissues. (A) Immunohistochemical staining of PLIN1 in liposarcoma tumor tissues and normal adipose tissues (magnification, x200), and (B) the IOD was measured for immunohistochemical positive staining. (C) Protein expression of PLIN1 was measured by western blotting, (D) histogram represents the gray intensity analysis of PLIN1. "P<0.05 vs. normal control group. PLIN1, perilipin-1; IOD, integrated optical density.

A
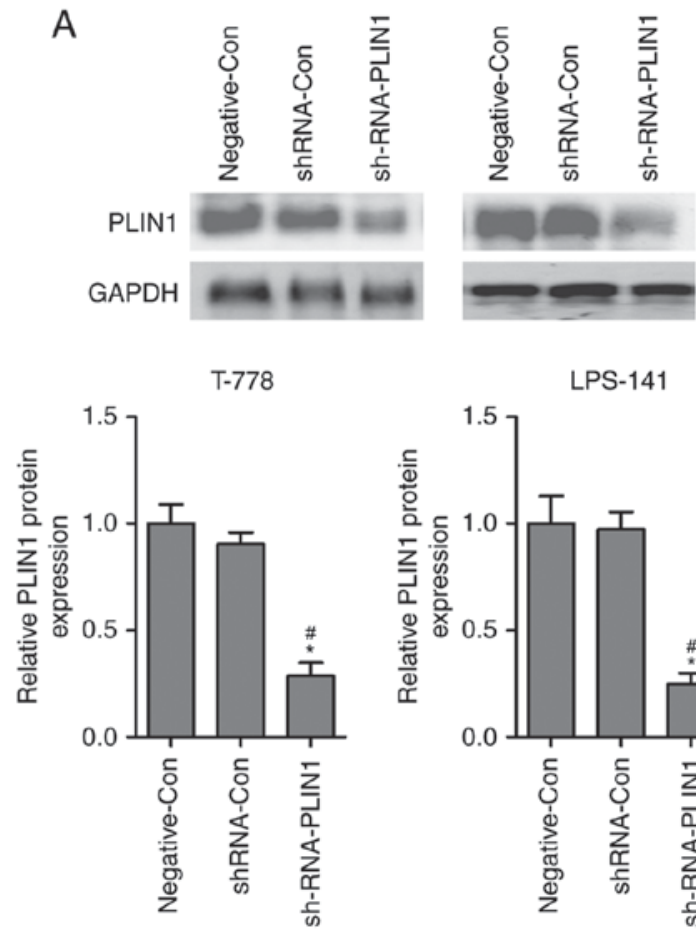

LPS-141
B
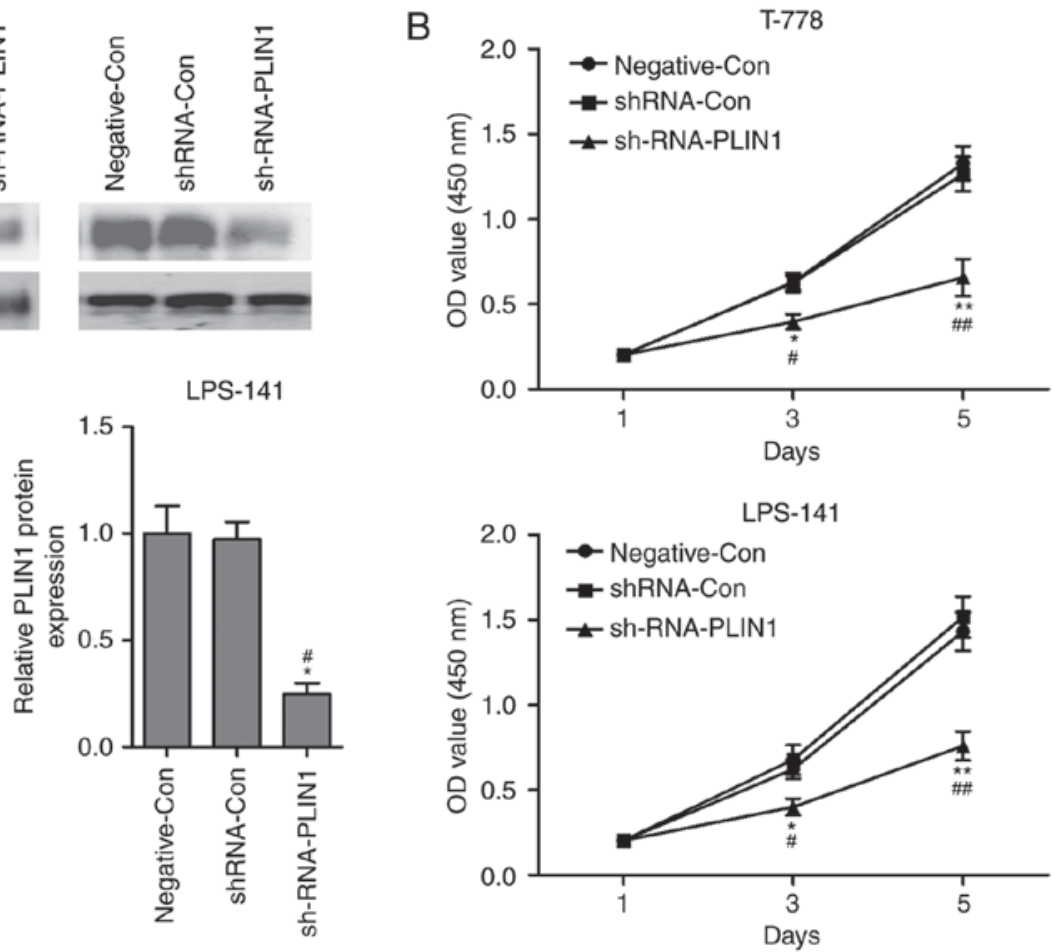

Figure 2. Silencing of PLIN1 suppresses proliferation of liposarcoma cells. (A) Following transfection with Negative-Con, siRNA-Con or siRNA-PLIN1, the protein levels of PLIN1 were detected using western blotting in T778 and LPS141 cells. (B) Following transfection with Negative-Con, shRNA-Con or shRNA-PLIN1, cells proliferation of T778 and LPS141 cells was monitored by CCK-8 assay at days 1,3 and $5 . \mathrm{n}=3$ in each group. ${ }^{*} \mathrm{P}<0.05,{ }^{* *} \mathrm{P}<0.01 \mathrm{vs}$. shRNA-Con group; ${ }^{\#} \mathrm{P}<0.05,{ }^{\# /} \mathrm{P}<0.01$ vs. Negative-Con. PLIN1, perilipin-1; Con, control; sh, short hairpin; OD, optical density.

arrest (2), so the cell cycle distributions were analyzed using flow cytometry to detect the effects of shRNA-PLIN1 on cell cycle arrest in T778 and LPS141 cells. It was identified that shRNA-PLIN1 significantly affected the cell cycle distributions, increasing the percentage of cells in $G_{1}$ phase and decreasing the percentage of cells in S phase. Notably, apoptosis was also induced in T778 and LPS141 cells transfected with shRNA-PLIN1.

In conclusion, the findings of the present study indicated that the expression of PLIN1 was altered in liposarcoma tumor 

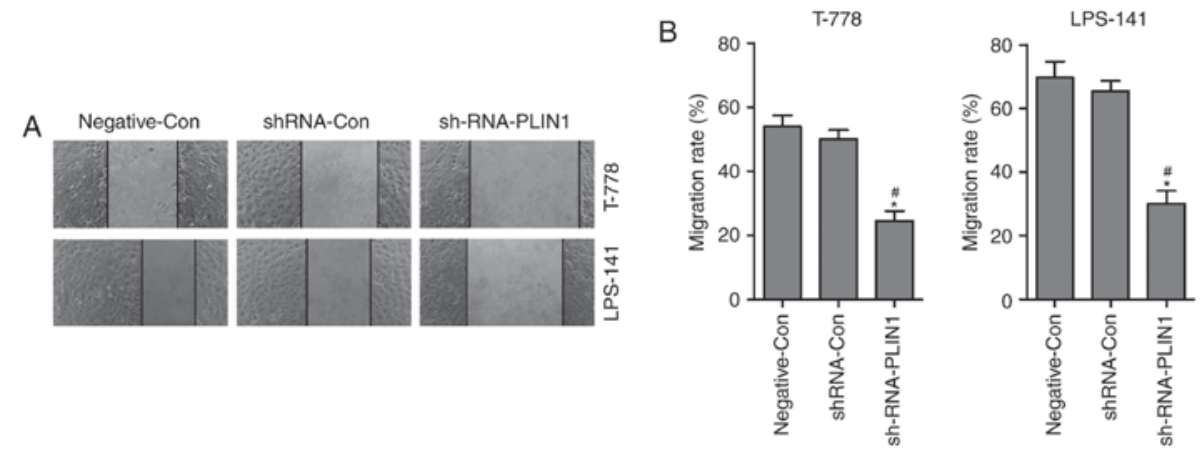

Figure 3. Silencing of PLIN1 suppresses migration of liposarcoma cells. (A) Following transfection with Negative-Con, shRNA-Con or shRNA-PLIN1, cell migration was determined by a wound healing assay, (B) histogram represents the migration rate. $\mathrm{n}=3$ in each group. ${ }^{*} \mathrm{P}<0.05$ vs. shRNA-Con group; ${ }^{\prime} \mathrm{P}<0.05$ vs. Negative-Con. PLIN1, perilipin-1; Con, control; sh, short hairpin.

A
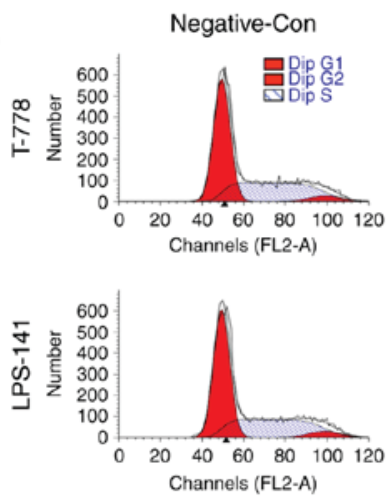

B

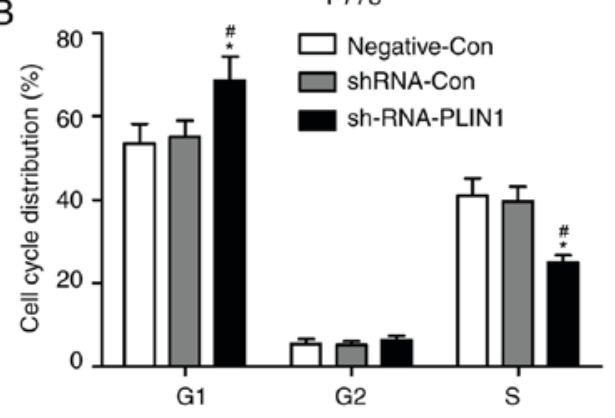

ShRNA-Con
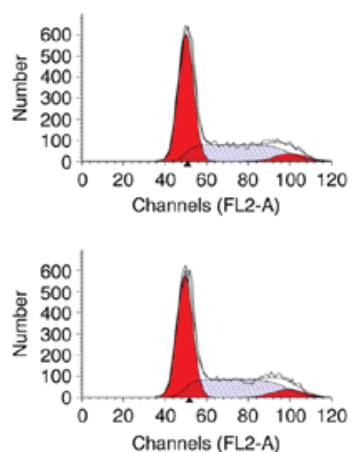

sh-RNA-PLIN1
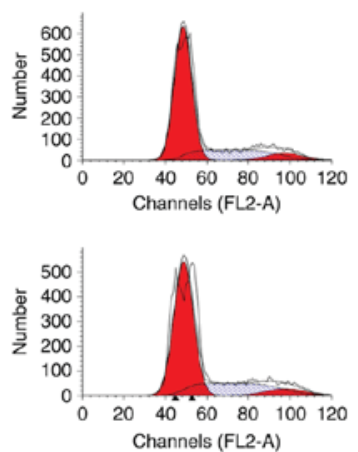

LPS-141

\section{$c$}

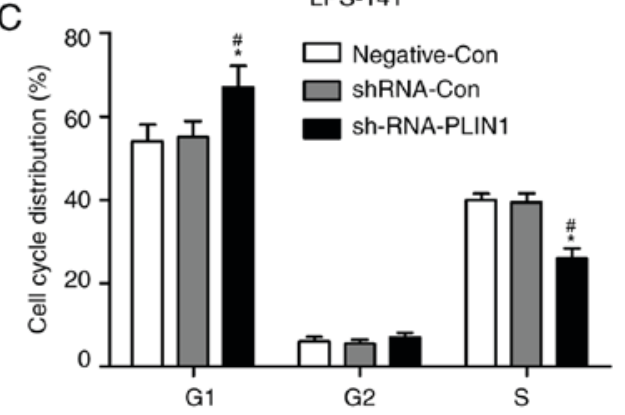

Figure 4. Silencing of PLIN1 induces cell cycle arrest of liposarcoma cells. (A) Following transfection with Negative-Con, shRNA-Con or shRNA-PLIN1, T778 and LPS141 cells were stained with propidium iodide to determine cell cycle distributions using flow cytometry. (B and C) Data as a histogram. $\mathrm{n}=3$ in each group. " $\mathrm{P}<0.05$ vs. shRNA-Con group; ${ }^{\text {"P }}<0.05$ vs. Negative-Con. PLIN1, perilipin-1; Con, control; sh, short hairpin.
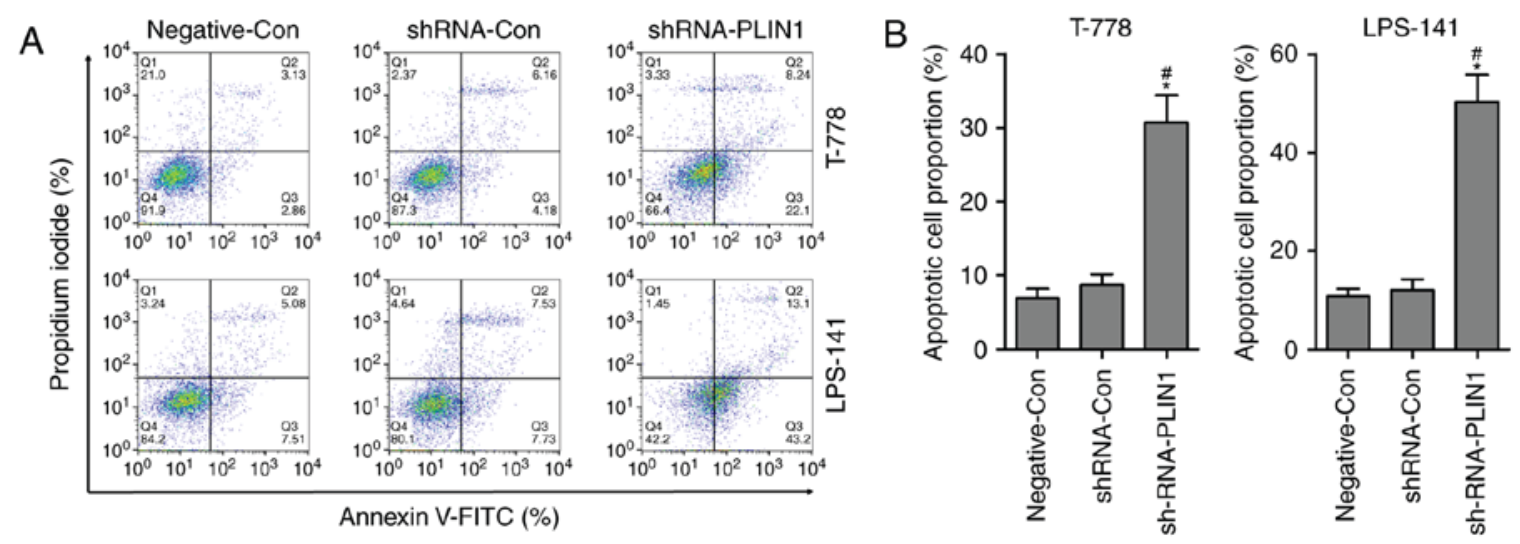

Figure 5. Silencing of PLIN1 induces apoptosis of liposarcoma cells. (A) Following transfection with Negative-Con, shRNA-Con or shRNA-PLIN1, T778 and LPS141 cells were stained with Annexin V-FITC and propidium iodide, and analyzed by flow cytometry. (B) Data as a histogram. $\mathrm{n}=3$ in each group. ${ }^{*} \mathrm{P}<0.05$ vs. shRNA-Con group; ${ }^{\text {PP }}<0.05$ vs. Negative-Con. PLIN1, perilipin-1; Con, control; sh, short hairpin. 
tissues, and preliminarily determined that PLIN1 was a key regulator of liposarcoma. In vitro experiments demonstrated that silencing of PLIN1 inhibited the proliferation and migration of liposarcoma cells by inducing $\mathrm{G}_{1}$ phase cell cycle arrest and apoptosis. However, PLIN1-associated signaling pathways were not involved in the present study, and PLIN1-induced apoptotic mechanisms need to be further investigated in in vivo and in vitro experiments.

\section{Acknowledgements}

The authors would like to thank the Department of Pathology of the People's Hospital of Rizhao for their technical support.

\section{Funding}

This work was supported by Shandong Medical and Health Science and Technology Development Program (grant no: 2016WS0329).

\section{Availability of data and materials}

All data generated or analyzed during this study are included in this published article.

\section{Authors' contributions}

The study was designed by LXM, YXZ and MLH. Literature research, data acquisition and data analysis were performed by XMZ, SYS, ZJD, QM, BCL and YWS. Histological examination was conducted by ZJD, QM, BCL and YWS. The manuscript was prepared and edited by LXM and YXZ. The manuscript was reviewed by LXM, YXZ and MLH. LXM, YXZ, MLH, XMZ, SYS, ZJD, QM, BCL and YWS approved the final version of the manuscript.

\section{Ethics approval and consent to participate}

The study was approved by the Ethics Committee of the People's Hospital of Rizhao (Approval Number: KYLL-20140903). Human samples were obtained with written informed consent from all patients.

\section{Patient consent for publication}

All patients provided consent for publication.

\section{Competing interests}

The authors declare that they have no competing interests.

\section{References}

1. Garg M, Kanojia D, Mayakonda A, Said JW, Doan NB, Chien W, Ganesan TS, Chuang LS, Venkatachalam N, Baloglu E, et al: Molecular mechanism and therapeutic implications of selinexor (KPT-330) in liposarcoma. Oncotarget 8: 7521-7532, 2017.
2. Wu J, Zhong D, Wei Y, Wu X, Kang L and Ding Z: Potassium channel ether a go-gol is aberrantly expressed in human liposarcoma and promotes tumorigenesis. Biomed Res Int 2014: 345678, 2014.

3. Franz H, Greschik H, Willmann D, Ozretić L, Jilg CA, Wardelmann E, Jung M, Buettner R and Schüle R: The histone code reader SPIN1 controls RET signaling in liposarcoma. Oncotarget 6: 4773-4789, 2015.

4. Azar AR, Weynand B, Daumerie C and Coche E: Metastatic liposarcoma of the thyroid gland. Br J Radiol 76: 750-752, 2003.

5. Tseng WW, Somaiah N, Lazar AJ, Lev DC and Pollock RE: Novel systemic therapies in advanced liposarcoma: A review of recent clinical trial results. Cancers 5: 529-549, 2013.

6. Westhoff CC, Mrozinski J, Riedel I, Heid HW and Moll R: Perilipin 1 is a highly specific marker for adipocytic differentiation in sarcomas with intermediate sensitivity. J Cancer Res Clin Oncol 143: 225-232, 2017.

7. Zhou C, Wang M, Zhou L, Zhang Y, Liu W, Qin W, He R, Lu Y, Wang Y, Chen XZ and Tang J: Prognostic significance of PLIN1 expression in human breast cancer. Oncotarget 7: 54488-54502, 2016.

8. Martinez-Botas J, Anderson JB, Tessier D, Lapillonne A, Chang BH, Quast MJ, Gorenstein D, Chen KH and Chan L: Absence of perilipin results in leanness and reverses obesity in Lepr (db/db) mice. Nat Genet 26: 474-479, 2000.

9. Souza SC, de Vargas LM, Yamamoto MT, Lien P, Franciosa MD, Moss LG and Greenberg AS: Overexpression of perilipin A and B blocks the ability of tumor necrosis factor alpha to increase lipolysis in 3T3-L1 adipocytes. J Biol Chem 273: 24665-24669, 1998.

10. Puri V, Ranjit S, Konda S, Nicoloro SM, Straubhaar J, Chawla A Chouinard M, Lin C, Burkart A, Corvera S, et al: Cidea is associated with lipid droplets and insulin sensitivity in humans. Proc Natl Acad Sci USA 105: 7833-7838, 2008.

11. Chen M, Wang H, Li X, Li N, Xu G and Meng Q: PLIN1 deficiency affects testicular gene expression at the meiotic stage in the first wave of spermatogenesis. Gene 543: 212-219, 2014.

12. Zhao X, Gao M, He J, Zou L, Lyu Y, Zhang L, Geng B, Liu G and Xu G: Perilipin1 deficiency in whole body or bone marrow-derived cells attenuates lesions in atherosclerosis-prone mice. PLoS One 10: e0123738, 2015.

13. Meyer MB, Benkusky NA, Sen B, Rubin J and Pike JW: Epigenetic plasticity drives adipogenic and osteogenic differentiation of marrow-derived mesenchymal stem cells. J Biol Chem 291: 17829-17847, 2016.

14. Jung YY, Kim HM and Koo JS: Expression of lipid metabolism-related proteins in metastatic breast cancer. PLoS One 10: e0137204, 2015.

15. Liang HF, Zhang XZ, Liu BG, Jia GT and Li WL: Circular RNA circ-ABCB10 promotes breast cancer proliferation and progression through sponging miR-1271. Am J Cancer Res 7: 1566-1576, 2017.

16. Seo JH, Jeong ES and Choi YK: Therapeutic effects of lentivirus-mediated shRNA targeting of cyclin D1 in human gastric cancer. BMC Cancer 14: 175, 2014.

17. Peng YT, Shi XE, Li ZQ, He X and Sun YM: Particularly interesting Cys-His-rich protein is highly expressed in human intracranial aneurysms and resists aneurysmal rupture. Exp Ther Med 12: 3905-3912, 2016.

18. Brasaemle DL, Barber T, Wolins NE, Serrero G, Blanchette-Mackie EJ and Londos C: Adipose differentiation-related protein is an ubiquitously expressed lipid storage droplet-associated protein. J Lipid Res 38: 2249-2263, 1997.

19. Kimmel AR, Brasaemle DL, McAndrews-Hill M, Sztalryd C and Londos C: Adoption of PERILIPIN as a unifying nomenclature for the mammalian PAT-family of intracellular lipid storage droplet proteins. J Lipid Res 51: 468-471, 2010.

20. Straub BK, Herpel E, Singer S, Zimbelmann R, Breuhahn K, Macher-Goeppinger S, Warth A, Lehmann-Koch J, Longerich T, Heid $\mathrm{H}$ and Schirmacher P: Lipid droplet-associated PAT-proteins show frequent and differential expression in neoplastic steatogenesis. Mod Pathol 23: 480-492, 2010.

21. Heid H, Rickelt S, Zimbelmann R, Winter S, Schumacher H, Dörflinger Y, Kuhn C and Franke WW: On the formation of lipid droplets in human adipocytes: The organization of the perilipin-vimentin cortex. PLoS One 9: e90386, 2014. 